\title{
Research on Trajectory Scheme with Large Impact Angle for Fire-and-Forget Anti-Tank Missiles
}

\author{
Wendong Zhao ${ }^{1, a}$, Min Gao ${ }^{1, b}$, Wei Huang ${ }^{1, b}$, Huimin Wang ${ }^{2, d}$ \\ ${ }^{1}$ Mechanical Engineering College, Shijiazhuang 050003, China; \\ ${ }^{2}$ Unit 61267, Beijing 101114, China. \\ a13315987059@163.com, bgaomin1106@sina.com, cbuaahuangwei@126.com, ${ }^{d} 274544208 @ q q . c$ \\ om
}

Keywords: anti-tank missile, angle constraints, guidance law, autopilot.

\begin{abstract}
In order to achieve the damage effect, when the anti-tank missile hits the target in the top attack model, both precision and terminal impact angle should be satisfied. Firstly, a kind of proportional navigation law with impact position and angle constraint was deduced. Then, the top attack trajectory of the anti-tank missile was designed, the missile flies out of the tube without control, the climb stage was completed by the index number regulation, the stage of glide at a certain angle of attack, at the end of the trajectory the guidance law which was deduced was adopted to satisfy the impact position and angular constraint. The required autopilots were also designed. The results of simulation showed that the trajectory scheme has strong effectiveness for the anti-tank missile to attack targets at different range.
\end{abstract}

\section{Introduction}

In order to improve the damage effect on main battle tanks, armored vehicles and non-armored targets, the trajectory of the new generation fire-and-forget anti-tank missile is flexible, The missile is able to achieve top-attack, terminal impact angle is proposed high demands while ensuring the accuracy of the missile. Firstly, a kind of proportional navigation law with impact position and angle constraint is deduced. Then, the top attack trajectory of the anti-tank missile is designed. The autopilots of the missile were also designed to control the missile flight stably. Finally verified the trajectory project can meet the tactical and technical requirements by simulation.

\section{A kind of proportional navigation law with impact position and angular constraints}

For proportional navigation law is a mature guidance law and is very easy to be implemented, it has got a wide range of engineering applications. Adopting a general proportional navigation law, although it can meet the requirement of miss distance, but it can not meet the requirement of the large drop-angle. Therefore, it was necessary to modify the proportional navigation to meet the requirement of large impact angle. For the target of rest or uniform motion, linear simplified dynamic model of missile guidance problems shown in Figure 1, where $y$ denotes the missile position, $a_{M}$ presents the missile acceleration.

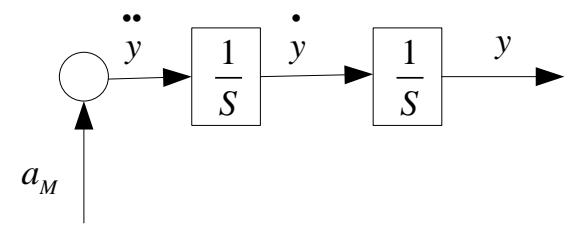

Fig. 1 Guidance law derived model diagram

Its state equation can be expressed as:

$$
\dot{x}=A x+B u
$$




$$
x=\left[\begin{array}{ll}
y & \dot{y}
\end{array}\right]^{T}, A=\left[\begin{array}{ll}
0 & 1 \\
0 & 0
\end{array}\right], B=\left[\begin{array}{ll}
0 & -1
\end{array}\right]^{T}, u=a_{M} .
$$

Because the terminal miss distance must be zero and meet angular constraint, the terminal constraint is expressed as: $x_{f}=\left[\begin{array}{ll}0 & \dot{y}_{f}\end{array}\right]^{T}$, The objective function is expressed as: $J=0.5 \int_{0}^{t_{f}}\left(u^{2} / \mathrm{t}_{g o}^{n}\right) d t$.

Among them: $t_{f}$ denotes total flight time, $t$ denotes the time the missile has flight, $\mathrm{t}_{g o}=t_{f}-t$ denotes the remaining flight time, From references[2,3,4], we know $t_{g o}=r / v_{r}, r$ indicates the relative distance between the missile and the target, $v_{r}$ presents the relative velocity of the missile and the target. According to reference [1], take advantage of Schwartz inequality, a guidance law with angle constraint can be deduced: $a_{M}(t)=K_{1}\left[y(t)+\dot{y}(t) t_{g o}\right] / t_{g o}^{2}+K_{2}\left[y(t)+\dot{y}_{f} t_{g o}\right] / t_{g o}^{2}$

In the assumption of small angle,

$$
y(\mathrm{t})=-q(t) v_{r} t_{g o} \quad(1), \quad \dot{y}(t)=-\dot{q}(t) v_{r} t_{g o}+q(t) v_{r} \quad(2), \quad \dot{y}_{f} \approx-v_{r} q_{f} \approx-v_{r} \theta_{f}
$$

Where: $q(t)$ denotes the sight angle, $\dot{q}(t)$ denotes the rate of sight angle; $q_{f}$ denotes the terminal sight angle; $\theta_{f}$ denotes the terminal desired drop-angle. The equations (1) $\sim$ (3) substitute into the guidance law can obtain: $a_{M}(t)=K_{1} v_{r} \dot{q}(t)+K_{2} v_{r}\left[q(t)-\theta_{f}\right] / t_{g o}$

The guidance law is composed by the item of proportional navigation and the item of terminal angular constraint.

When $n=0: a_{M}(t)=4 v_{r} \dot{q}(t)+2 v_{r}\left[q(t)-\theta_{f}\right] / t_{g o}$ is the general optimal guidance law, When $n>0$ : Because the existence of $\lim _{t \rightarrow t_{f}}\left(u^{2} / t_{g o}^{n}\right), \lim _{t \rightarrow t_{f}} u=0$. It means that when the missile hit the target the acceleration command is zero, the missile cannot resist wind and other external interference factors and it will have great impact on miss distance. As $3 \sim 6$ is the scope of coefficient of the proportional navigation, the value of $n$ is in the range of $-0.5 \sim 1$ in special engineering application.

\section{The design of anti-tank missile top-attack trajectory}

The power unit of the anti-tank missile is a double pulse solid rocket engine. The $I$ pulse is used to boost the missile flies out of the launchers. After the missile flied to a certain distance from the launch position, the II pulse of the engine is ignited to provide power for the missile to have controlled flight. Because the distance of the recognition of the homing system is less than the maximum range of the missile, the inertial midcourse guidance and the infrared terminal guidance are applied to the missile. The missile flies to a predetermined position after the midcourse guidance to ensure the seeker intercept the target reliability and improve guidance accuracy. Under the existing engineering conditions, in order to meet the relevant requirements of the tactical and technical indicators, the ballistic scheme was designed to be: the stage of emission without control, the stage of climbing rely on procedures, the stage of driving down trajectory rely on procedures and the terminal guidance.

\subsection{The stage of emission without control}

The segment is the interval between the ignition of $I$ pulse and ignition of II pulse. The missile lunched upwards at a certain degree of attitude angle, the missile flied out of the launchers, wings unfolded, missile flied forward with rotating, but the fins of the missile were not work.

\subsection{The stage of climbing rely on procedures}

To increase the range and achieve large drop-angle, the flight height of the missile must be improved. The segment of climbing relied on procedures to control, its variation of pitch angle and angle of attack is based on indicators of performance such as overload, range and so on. The duration of the segment is from the ignition of II pulse to the begin of gliding flight (T2). After the ignition of II pulse, the missile was speed up. The flight control system controls the gas rudder and the air rudder 
deflection, the missile no longer rotates, using the procedures to control the angle of attack in accordance with exponentially $\alpha=\alpha_{0} e^{b t}$ in the longitudinal plane to climb. Among them: $\alpha_{0}$ presents the initial angle of attack for the climb segment, $b$ is a constant value, $t$ is the time to climb. To make missile climbs rapidly, $\alpha_{\max }$ is the maximum allowable value of the angle of attack in this segment. The calculation equation of procedures pitch angle in this stage is:

$$
\phi_{c x}(k)=\phi_{c x}(k-1)+\alpha_{\max }\left[1-e^{-\left(t-T_{1}\right) / T_{2}}\right] \cdot \Delta T, \phi_{c x}(0)=\phi_{c x}\left(T_{1}\right) .
$$

Among them: $\phi_{c x}$ denotes the procedure pitch angle, $\Delta T$ denotes the sampling time.

\subsection{The stage of driving down trajectory rely on procedures}

After the missile climbed to a certain height, in order to hit the target in the top attack model, the pitch angle of the missile must be reduced. This stage is from the end of stage of climb relied on procedures (T2) to the beginning of terminal guidance (T3). Its purpose is to increase the range, search and acquire targets in the process. The calculation equation of procedures pitch angle in this stage is:

$$
\phi_{c x}(k)=\phi_{c x}(k-1)+\alpha_{p p} \cdot \Delta T \phi_{c x}(0)=\phi_{c x}\left(T_{2}\right)
$$

The value of $\alpha_{p p}$ was determined by the distance between the missile and the target.

\subsection{The terminal guidance}

After the seeker acquired the target and the seeker worked in an ideal condition. The seeker can normally output the rate of sight angle, and the sight angle. The guidance law of longitudinal plane becomes:

$$
a_{M}(t)=K_{1} v_{r} \dot{q}(t)+K_{2} v_{r}\left[q(t)-\theta_{f}\right] / t_{g o}
$$

\section{The autopilot design}

The dynamics of the anti-tank missile is changing under the influence of factors such as velocity, the center of gravity, the center of pressure and so on. In accounting with the influence of quiver of missile at the launching time and the uncertain disturbance during the process of flight, the autopilot is needed to improve the dynamics of the missile and reduce the negative impact. The control instruction of the missile is different during the flight. The attitude angle control instruction is adopted during the midcourse guidance and in the terminal guidance the missile is controlled by acceleration instruction. Based on the principle that guidance law and autopilot must match each other, different kinds of autopilot must be adopted during the flight. The attitude autopilot (Fig.2) is adopted in midcourse guidance and acceleration autopilot (Fig.3) is adopted in terminal guidance .

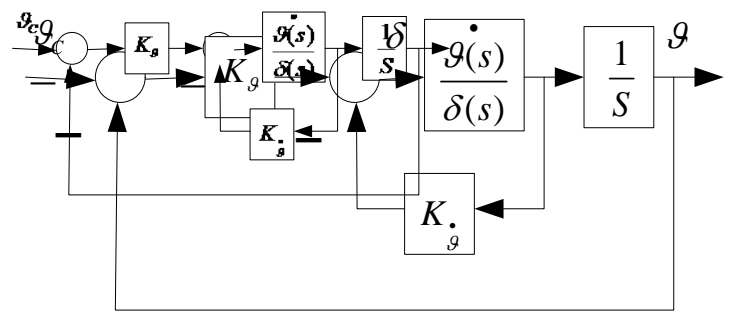

Fig. 2 Attitude autopilot block diagram

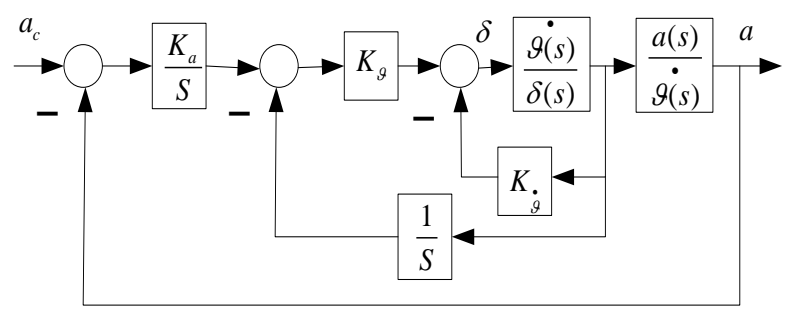

Fig. 3 Acceleration autopilot block diagram 


\section{Simulation and Analysis}

In order to verify the effectiveness of the scheme of top -attack designed for anti-tank missile, combining with related parameters of anti-tank missile to have a simulation. The conditions of the simulation was: the distance of a fixed target and the launch point was respectively set to $1000 \mathrm{~m}$, $1500 \mathrm{~m}, 2000 \mathrm{~m}, 2500 \mathrm{~m}$. The angular constraint $\theta_{f}$ is $-45^{\circ}$. The simulation results were as follows:

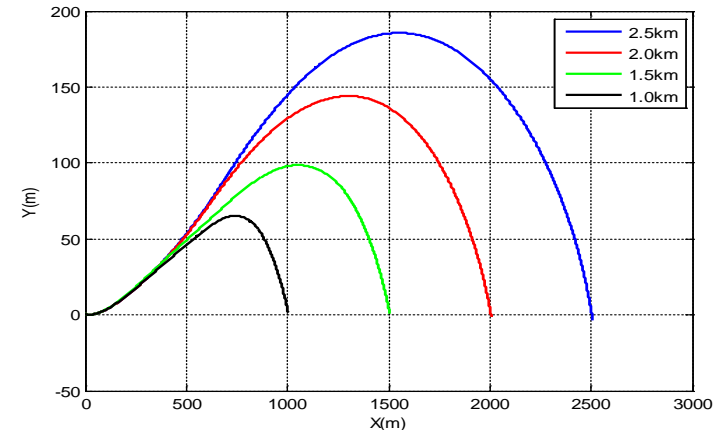

Fig. 4 Range and height curve

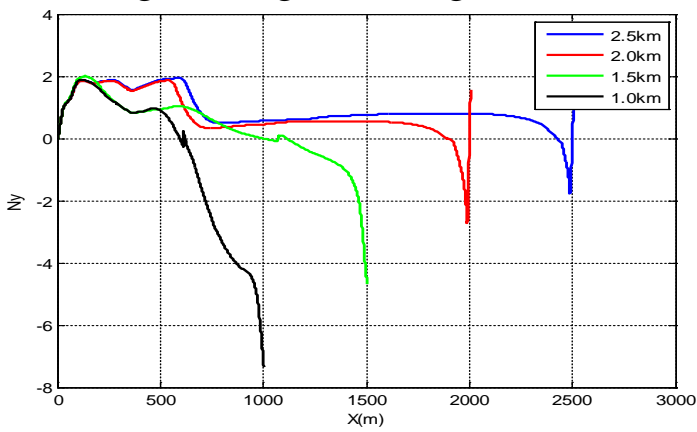

Fig. 6 Range and overload curve

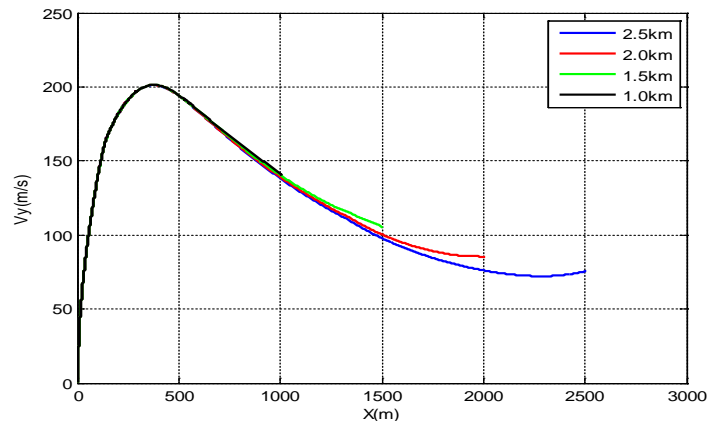

Fig. 5 Range and speed curve

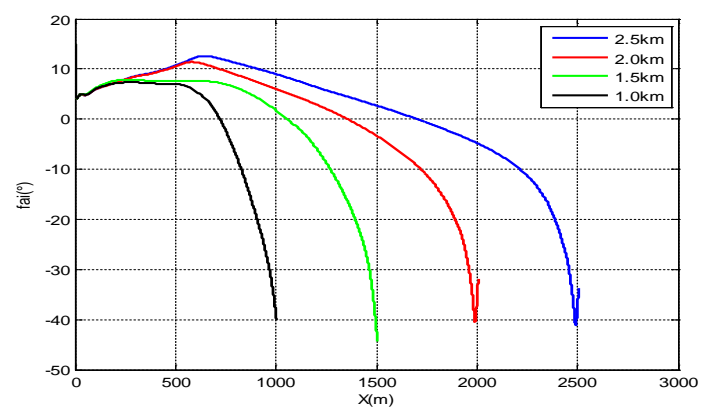

Fig. 7 Range and angle curve

Data table of different trajectory

\begin{tabular}{cccccc}
\hline Range $(\mathrm{km})$ & Highest point $(\mathrm{m})$ & $\begin{array}{c}\text { Landing } \\
\text { point speed }(\mathrm{m} / \mathrm{s})\end{array}$ & $\begin{array}{c}\text { Impact } \\
\text { angle }(\circ)\end{array}$ & Maximum overload $(\mathrm{g})$ & $\Delta z(\mathrm{~m})$ \\
\cline { 1 - 4 } 2.5 & 185.6 & 75.87 & -41.03 & 1.937 & 0.01 \\
2.0 & 144.0 & 85.5 & -40.52 & -2.72 & -0.16 \\
1.5 & 98.59 & 105 & -44.47 & -4.68 & 0.48 \\
1.0 & 65.27 & 140.8 & -40.14 & -7.3 & -0.5 \\
\hline
\end{tabular}

The simulation results showed that:

(1) The height of the trajectory increased with the increasing of the range. The height range of the trajectory is from $65.27 \mathrm{~m}$ to $185.6 \mathrm{~m}$.

(2) The velocity of the missile decreased with the increasing of the range. At the range of $1000 \mathrm{~m}$, the speed of the missile at the landing point is $140.8 \mathrm{~m} / \mathrm{s}$. At the range of $2500 \mathrm{~m}$, the speed at the landing point is $75.87 \mathrm{~m} / \mathrm{s}$.

(3) The miss distance of the missile is very small and the terminal impact angle meets the requirements. Both the maximum miss distance and the maximum angular deviation occur at the range of $1000 \mathrm{~m}$. The value of maximum miss distance is $0.5 \mathrm{~m}$ and the value of maximum angular deviation is $4.86^{\circ}$.

(4) Because the calculation error of the remaining flight time, the overload of the terminal guidance increased rapidly and failed to convergence. In engineering practice, we can set the guided instruction to be zero at a certain distance from the target to meet the requirement of terminal overload. 


\section{Conclusions}

The top-attack trajectory of fire-and-forget anti-tank missile was studied. By designing the stage of emission without control, the stage of climbing rely on procedures, the stage of driving down trajectory rely on procedures and terminal guidance phase. The autopilot was also designed for the missile. The anti-tank missile can attack the target at different range in the top-attack model. The trajectory scheme can satisfy with the tactical indicators and technical indicators, and has a certain value of engineering application.

\section{References}

[1] Paul Zarchan. Tactical and strategic missile guidance [M]. 5th ed, Virginia: AIAA Inc,2007.

[2] Ryoo C K, Cho H, Tahk M J. Optimal guidance laws with terminal impact angle constraint [J]. Journal of Guidance, Control, and Dynamics, 2005, 28(4):724-732.

[3] Ryoo C K, Cho H, Tahk M J. Time-to-go weighted optimal guidance with impact angle constraints [J]. IEEE Transactions on Control Systems Technology, 2006, 14(3):483-492.

[4] Ryoo C K, Cho H, Tahk M J. Closed-form solutions of optimal guidance with terminal impact angle constraint. [C]. Proceedings of IEEE Int Conf Control Application. Istanbul, Turkey: [s.n], 2003: 504-509.

[5] Zhenduo Song, Qing Diao, Wenxu Xu. Ballistic model and simulation of a portable antitank missile steering to hit on top of the tank at terminal trajectory[J]. Acta Armamentarii, 2005, 26(2):209-214..

[6] Jingung Shi, Zhongyuan Wang, Xiaobing Cao. Design of glide trajectory for glide range-extended projectile[J]. Journal of Nanjing University of Science and Technology, 2007, 31(2):147-150..

[7] Jian Chen, Huibin Gao, Jin Guo. Analysis of “javelin” anti-tank missile guidance mode[J]. Infrared and Laser Engineering, 2014, 43(6):1777-1781..

[8] Qi Liu, Jun Yang. Different guidance information with terminal guidance law design of the angular constraints[J]. Computer Simulation, 2013,4(4):33 36

[9] Yajun Tian, Gang Zhou, Hongqiang Wang. Research on trajectory of guided penerating bomb[J]. Tactical Missile Technology, 2011(5):50-53..

[10] Qiuqiu Wen, Qunli Xia, Zaikang Qi. Research of trajectory scheme with large impact angle for imaging guided AGM[J]. Journal of Projectiles, Rockets, Missiles and Guidance, 2010, 30(3):8-10.. 